\title{
Support Mobility in the Global Internet
}

\author{
Lixia Zhang \\ UCLA \\ lixia@cs.ucla.edu
}

\author{
Ryuji Wakikawa \\ Toyota Infotechnology Center \\ ryuji@us.toyota-itc.com
}

\author{
Zhenkai Zhu \\ UCLA \\ zhenkai@cs.ucla.edu
}

\begin{abstract}
Today's technology trend indicates that billions of handheld gadgets as well as other types of mobile devices will be coming online in the next few years. While the existing Internet mobility standards, namely Mobile IP, is waiting for a wide adoption, cellphone networks are providing the ubiquitous mobility services on a global scale as of today. They have also promoted IP core network architecture and adopted Proxy Mobile IPv6, an extension to Mobile IP, for their mobility service. There is an open question regarding whether the Internet would, or would not, require significant architectural changes to provide universal mobility support at a scale that is likely to go far beyond the scale and scope of today's cellular telephone services. In this paper, we examine the fundamental differences between the mobility service models provided by Internet and cellphone systems. We argue that decoupling network access control from mobility support can provide an architecturally promising direction for scalable and decentralized mobile communications, and that designing mobility support outside the global routing system can offer an overall best tradeoff as measured by flexibility, manageability, and scalability of the resulting systems.
\end{abstract}

\section{INTRODUCTION}

In a research proposal submitted to the National Science Foundation ten years ago, we speculated that "within the next few years mobile and wireless access to the Internet will very likely become the norm, rather than the exception as we see today", thus efforts were needed to develop an infrastructure that "provides support for anytime, anywhere, on any platform access to the Internet" [1]. This prediction came true over the last few years. Today one can state with confidence that there are probably more people who access Internet via mobile devices of one type or another (e.g. PDAs, cellphones) than the number of users on stationary hosts with wired connectivity. In addition to mobile hosts, there have also been mobile networks on the trains, and on airplanes as pioneered by Boeing's Connexion service ${ }^{1}$. Furthermore, the future may also see mobile sensors performing monitoring duties.

What would be the best way to provide global scale ubiquitous mobility support for a essentially unlimited number of mobile devices with unknown future applications? Multiple answers exist today. Mobile IP is an Internet standard pro-

\footnotetext{
${ }^{1}$ Although Connexion service terminated, there have been new trials on providing Internet access on airplanes.
}

tocol to support host mobility; over the last few years IETF has also been actively developing new standards for supporting network mobility. At the same time, the largest global mobility support today is provided by cellular networks, using a service model that bundles together the device control, network access control, and mobility support.

In this paper we articulate that the mobility support for the global Internet should take a fundamentally different model from the one used by today's cellular networks. We first discuss the design goals and requirements in Section 2. In section 3 we show that all mobility support designs involve the same three basic components: identifier, IP addresses, and a mapping system in between. In Section 4 we make several observations from the existing development and deployment efforts. Section 5 discusses new opportunities that are brought by opening up the platforms of hand-held devices as well as several open issues in mobility support. We conclude the paper in Section 6.

The contribution of this paper is 2-fold. First, it provides an analysis of mobility support design space and shows by example that different mobility designs are simply different realizations of the same three basic components, together with a discussion on general direction for future global mobility support development. Second, it puts forth a position that mobility support should be designed outside the global routing system, and that mobile nodes' network access control and mobility management should not be bundled together as in today's cellular networks.

\section{DESIGN GOALS}

To conjecture the future directions of mobility support, one needs to start with an ordered list of the fundamental design goals. As Clark argued in a discussion on the design goals for the Internet architecture [2], "It is important to understand that these goals are in order of importance, and an entirely different network architecture would result if the order were changed." What should be in the set of the fundamental design goals for future mobility support in Internet? And how should one order them?

To answer these questions, one may take a look at the relatively short history of the Internet itself. What enabled its explosive growth? One can identify a few important enablers, the open access (anyone can connect to Internet at a low cost), open architecture, and distributed management. Of course the success of Internet is fueled by the advancement of computing technologies and innovations in applications, however the open access eased interconnections of all interested parties, the open architecture offered a low 
threshold to enter for new users and new innovations, and the absence of central control or central authority removed potential constraints on its growth.

Based on what we learn from the Internet history, we would like to put forth the following requirements at the top of the list of the design goals for future mobility support. First, effectively connecting mobile devices to Internet should be of the first and foremost importance, above any other desired goals such as guaranteed service quality or ease of accounting. Second, the solutions should be able to support mobility for an unlimited number and a large variety of mobile devices in a cost effective way. Furthermore, increasing numbers of mobile devices will inevitably bring in new mobile applications that we may not envision today, which suggests that it would be best to decouple mobility support from applications above it.

\section{BASIC COMPONENTS IN MOBILITY PROTOCOLS}

Mobility support can be provided through multiple different ways. The basic question is how to make data reach a moving receiver (a mobile in short; in this paper we do not distinguish between mobile nodes and mobile subnets). Whoever sending data to a mobile must be able to identify the receiver via a piece of stable information, where "stable" means that the information does not change as the mobile moves. However if the sender's knowledge about the mobile does not change while the mobile moves, some means must exist to bind that unchanging identifier of the mobile with its dynamically changing location. Locations on Internet are represented by IP addresses.

The above intuitive reasoning leads to the following observation: mobility support essentially involves three basic components: a stable identifier for a mobile, an IP address of the mobile's current location, and a mapping between the two. In the rest of this section we show that different mobility support designs are simply different ways to choose mobile identifiers and different approaches to provide mapping between the identifiers and the mobiles current IP addresses.

\subsection{Routing and Mapping}

All existing mobility support designs can be broadly classified into two basic approaches. The first one is to support mobility through dynamic routing. In such designs, a mobile keeps its IP address regardless of its location changes, thus the IP address can be used both to identify the mobile and to deliver packets to it. As a result, such designs do not require an explicit mapping function. Rather, the routing system must continuously keep track of mobiles' movements and reflect their current positions in the network on the routing table, so that at any given moment packets carrying the (stable) receiver's IP address can be delivered to the right place. The Connexion service [3] took this approach, where BGP is used to propagate airplane location updates. Naturally the flying airplanes generated frequent BGP updates, which were propagated throughout the Internet.

Supporting mobility through dynamic routing is conceptually simple as it does not require a mapping function; it can also provide robust and efficient routing, assuming that the routing system can keep up with the mobile movements. However, because the whole network must be informed of every movement by every mobile, this approach is feasible only in small scale networks with a small number of mobiles; it does not scale well in large networks or for large number of mobiles.

The second approach to mobility support is to provide a mapping between a mobile's stable identifier and its dynamically changing IP address. Instead of notifying the world on every movement, a mobile only needs to update a single binding location about its location changes. Both the cellular networks and the Mobile IP design take this second approach, so do a number of other mobility support protocols, for example Proxy Mobile IP [5] and HIP [6].

Several of the mobility support designs provide the mapping function at IP layer, where both the identifier and the current location of a mobile are represented by IP addresses. In this approach, the IP address which is used as a mobile's identifier points to a location which keeps track of the IP address of the mobile's current location. Such designs offer an advantage of hiding the mobility from correspondent nodes through one level of indirection. When a correspondent node sends packets to an IP address which is a mobile's identifier, the packets will be delivered to the location where the mapping information of the mobile is kept, so that the packets can be delivered to the mobile's current location via either encapsulation or destination address translation.

Although this one level of indirection at IP layer makes mobility transparent, it has a potential side effect of introducing triangle routing: the path taken by the packets via the mapping point can be much longer than the direct path between the correspondent and the mobile's current location. As increasing numbers of mobile devices are connected to Internet, some mobility solutions have opted to expose mobility to both ends and let them communicate directly. One common approach taken by these protocols is to use DNS for the mapping function to keep track of mobiles' current locations. Mobiles use dynamic DNS updates to keep their DNS servers updated with their current locations.

\subsection{Basic Components in Different Designs}

In the rest of this section we examine a few representative mobility systems to show how the three basic mobility support components, namely mobile identifier, mobile's current address, and the mapping in between, are implemented in each system. One can see that different systems only differ in their choices of where to place these components, as shown in Figure 1.

In cellular networks, each cellphone can be uniquely identified through its SIM card, which essentially provides secure identification. The location of the mobile is represented by the Serving GPRS Support Node (SGSN) the mobile is connected to. In a general packet radio service network (GPRS), A Gateway GPRS Supporting Node (GGSN) carries out a role equivalent to the Home Agent in Mobile IP. The cellphone's home service provider keeps the mapping of its number and its location at the HLR (Home Location Register).

In Mobile IP (MIP), every mobile node is identified by its Home Address, while its current location is represented by its Care-of-Address. The Home Agent keeps the mapping between these two and hides the mobility of mobile from the rest of the world. To mitigate Mobile IP's problems of triangle routing, Global HA-HA [10] introduces a distributed extension of Mobile IP. Instead of using a single Home Agent for each mobile, Global HAHA utilizes a set of cooperating Home Agents that are distributed over the Internet. The 

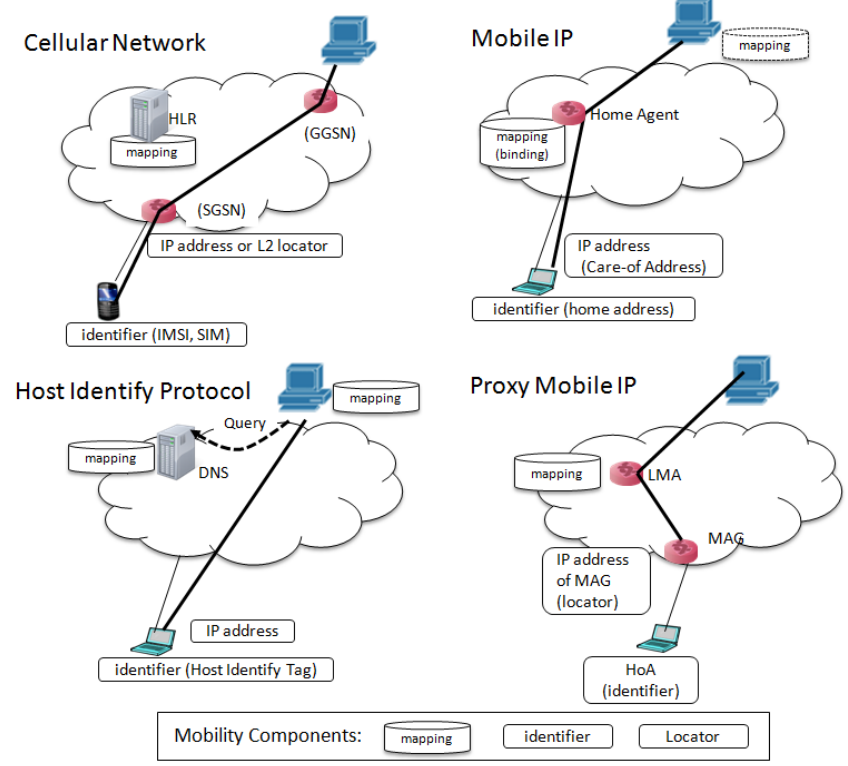

Figure 1: Components in Different Systems

mapping information is distributed among the Home Agents. All the Home Agents announce the same home subnet prefix to Internet, so that when a correspondent sends packets to a mobile, the packets will reach the nearest Home Agent, which then forwards the packets to the Home Agent that is closest to the mobile.

As a protocol favored by cellular operators, Proxy Mobile IPv6 [5] can be considered another extension to Mobile IP that frees a mobile from performing any mobility-related signaling. Instead, the network takes the responsibility for managing IP mobility on behalf of the mobile within a single operator's network. Each mobile retains its IP address when it roams within its domain (a network that uses PMIP for mobility support), and thus this address is equivalent to the Home Address in MIP, i.e. the identifier of the mobile. Mobile Access Gateway (MAG) monitors each mobile moving in and out its local access link and sends updates to Local Mobility Anchor (LMA). The IP address of MAG is used to reach the mobile, and LMA keeps the mapping between the Home Address of the mobile and the address of serving MAG.

Except cellular networks, all the above mentioned solutions provide mapping at IP layer. Routing based mobility support also work at IP layer but eliminates the need for mapping. With the requirement that all the routers in the network keep track of all mobiles locations all the time, mobile nodes or mobile subnets keep their IP addresses unchanged while moving, hence their IP addresses can be used both to identify destinations and to route to them.

The Host Identity Protocol [6] puts the mapping outside the IP layer. HIP assigns each host a cryptographic-based identifier which is totally independent from its IP address of the current location. A mobile updates its DNS server when moving to a new address. DNS is used to provide the mapping between the identifier and up-to-date IP address for a mobile. HIP may also use a dedicated server other than DNS, known as Rendezvous Point [7]. Mobile.Me [4], a quasi mobility service provided by Apple Inc. for MAC users, also uses DNS to provide mapping between the identifier and the current IP address of a mobile, but with a different identifier choice from HIP and with additional functions including data encryption and NAT traversal.

\section{OBSERVATIONS FROM EXISTING MOBILITY SYSTEMS}

In this section we make several observations from the existing mobility designs and services. We first discuss the current practices of access control and mobility management in cellular network and Internet, and then the relations between routing and mobility support.

\subsection{Access Control and Mobility Management}

Providing Internet connectivity to a mobile means two things: the mobile is connected to Internet, and it can be reached by any correspondent. The former involves access control, and the latter mobility management. Below we discuss how these two functions are implemented in cellular network and Internet, respectively.

\subsubsection{Cellular Networks Model}

Cellular networks implemented both functions in a combined way. First, each cellphone is made uniquely identifiable through its SIM card given by its home service provider. Second, when a cellphone $C$ wanders into the territory of a foreign provider, through a global number database the foreign provider can find $C$ 's home service provider. If the latter has contractual relations with the former, then $C$ can be granted network access. Third, when someone makes a call to $C$, the call is first routed to the home service provider and then redirected to $C$ 's current location. Here we note 3 essential pieces in this mobility support design: (1) the unforgeable identity of the cellphone, (2) the global database to find the home provider, and (3) the duel role of the home service provider: not only it performs mobility management for the cellphone, it also keeps the accounting book with foreign providers who grant the mobile device access to the foreign network resources. In other words, the access control and mobility management are bundled together as one service offer.

Another related point in cellular networks' mobility support is the clear distinction between local and global mobility, defined as whether a mobile node moves within a provider's network or across different providers' networks, respectively. One basic assumption about mobility in cellular networks is that a cellphone spends most of the time within the coverage of its home service provider. Interprovider roaming requires the commercial agreement between providers. Proxy Mobile IP that has been adopted by the future cellular core network EPC, Evolved Packet Core [11], closely follows the mobility management model of today's cellular networks. PMIP clearly separates local mobility (roaming within home provider's network) from global mobility (roaming between providers). Since PMIP installs all the mobility support in the network, a mobile does not need to do anything when it moves. On the other hand, the mobile cannot get connected in any network other than its home service provider and its provider's roaming parters networks. Given their tight control over exact where a mobile may be able to obtain network access, one may speculate whether the current cellular networks' control structure is adequate to support global mobility in near future. 


\subsubsection{Internet Model}

As we discussed earlier, IP mobility support concerns only the mobility management, assuming mobiles are already connected to Internet. Today's mobile laptops obtain Internet access as they go. It is up to individual devices and individual networks to decide whether to, and how to, grant network access to visiting mobile devices. Due to fundamental differences between cellular networks and Internet, the cellular networks' mobility support model is simply not applicable here. First of all, today's moving hosts in general do not have an unforgeable identity. Although it is technologically feasible to assign each host a cryptographic-based identifier, as the work done by the IETF HIP working group [14], that is not the common practice today. Thus moving laptops miss a fundamental component to mimic the access model used by cellphones. Second, different from cellular service market where a small number of major providers dominate (who also interconnect), there exist a large number of Internet service providers and most of them do not have direct interconnectivity; contractual relations only exist between topologically interconnected ISPs.

Consequently, Internet access control practice takes an entirely distributed model. Although almost everyone has an Internet service provider who provides Internet connection at home or in office, in general such "home Internet service providers" cannot, and do not, get involved in granting access to a laptop in a foreign network (except special cases where the home provider also provides hot-spot access service, such as T-mobile). Instead, individual mobile hosts gain their own Internet access right from foreign networks they move into. A typical example of this situation today is the Internet access provided at airports and hotels. Possible arrangements include foreign networks providing free Internet access (which is the case in many airports and hotels), or providing access for a fee. In the latter case, the payment process leverages on the existing credit card system, rather than assuming any verifiable identify of the mobile itself. ${ }^{2}$

Although global Internet mobility support is not yet realized today, Mobile IP represents an able candidate protocol to support mobility in global scale. A mobile node obtains Internet access as it goes, and receives mobility support from its home agent (which can be provided by any party on Internet that offers MIP's Home Agent service). The mobile node can move around anywhere on Internet with mobility support, irrespective of the relation between its home, if it has one, and foreign providers.

\subsubsection{Mobility Support in the Future}

Which one of the two models described above would be more likely to see wider adoption as time goes? Although no one can see clearly the crystal ball of the future, we may speculate based on the pros and cons of the two existing mobility service models.

The tremendous success of cellular market speaks loudly that the current cellular service model is a viable one, and is likely to continue for foreseeable future. Compared to

\footnotetext{
${ }^{2}$ One may argue that, if the foreign network access is not free, then an external database, hosted by the credit card system, indeed exists in order to handle the payment. However it may be considered a feature to be able to leverage on an existing database which already solved its user identification and authentication problems, instead of maintaining a separate one solely for mobility support.
}

the Internet model of separate access control and mobility management, however, the cellular network model imposes more stringent requirements on the mobiles (e.g. each one must have a SIM card), on the networks (e.g. contractual relations between each home and foreign network pair that a mobile may roam into), and on the global coordination (e.g. the global database that holds the complete list of cellphone identities with associated home service providers).

Internet access control practice, on the other hand, has no universal requirement on mobile devices, no assumptions about relations between providers, and no need for any global database to do lookup. Generally speaking, Internet mobile users do not have an equivalent "home service provider" as cellphone owners do. In fact, many people get Internet access as they go, without requiring explicit mobility support. At the same time, Internet mobility service practice also allow a diverse set of implementations whose mobility support goals span a wide range of spectrum from uninterrupted communication on the move to connection re-establishments after movements. Separating access from mobility not only makes each one simpler, but also provides the flexibility of allowing each one to be provided through multiple different means.

Thus, when one speculates a general access control model for the future where potentially orders of magnitude more mobile devices will need to get Internet access in foreign networks, although both models may co-exist as they do today, the Internet model, i.e. decoupling mobility support from access control, seems a much simpler way to move forward with. Besides, the Internet model's open and distributed nature already enabled mobility being added in various different forms, requiring no significant architectural changes in order to support mobility in an extremely large scale.

\subsection{Routing and Mobility Support}

Network routing and mobility support seem coupled in at least two possible ways. First, there have been designs and even trial implementations of mobility support through dynamic routing. After all, the sole purpose of network routing is to handle dynamic changes, would routing-based mobility support be a promising direction in the future? Second, in the last few years we have seen a number of proposed solutions to scaling global routing system that, remotely, seem resembler certain features of mobility solutions. In this section we articulate the relations between routing and mobility support.

\subsubsection{Scalability with Routing-Based Solutions}

Connexion [3] was the first trial of routing-based mobility support in the global Internet. Connexion assigned a /24 prefix to each airplane. Each airplane announced its prefix from its new attachment points during the flight, so that the routing system could effectively deliver data to its moving locations. However rapid movements of multiple airplanes led to a large amount of BGP updates being propagated throughout Internet, which raised the scalability concern about using dynamic routing to support mobility, especially in large scale networks. As the number of mobile entities grows, this approach becomes increasingly infeasible.

WINMO [9] is another network routing-based mobility support design. Like Connexion, WINMO also assigns each mobile subnet a stable prefix, however WINMO made the BGP update overhead for mobile networks orders of magni- 
tude lower than that of Connexion by the following two approaches. First, WINMO uses various heuristics to reduce the propagation scope of routing updates caused by mobile movements. Consequently, not every router may know all the mobiles current locations. Resolving this issue led to the second, and more fundamental, approach taken by WINMO: adoption of the basic idea from MIP. WINMO assumes that each mobile subnet is assigned a prefix out of a small set of well defined mobile prefixes. These mobile prefixes are announced by a small set of aggregation routers which keep track of the moving subnets. Thus one may view WINMO's aggregation routers as playing the role of Home Agents in MIP. WINMO design shows a supporting evidence that relying on dynamic routing alone for mobility support does not scale in large networks, but that the combination of Home Agent concept with network routing can potentially lead to a viable approach to global mobility support.

\subsubsection{Mapping in Mobility Support and in Routing Scalability}

Since early 2007 the Routing Research Group (RRG) under Internet Research Task Force has been working on a scalable routing architecture [15]. Although the design details are yet to be nailed down, most proposals that have been presented to RRG take on the map-n-encap approach [8]. To effectively scale down the routing system means to remove certain entries in today's routing tables, without losing reachability to any destination networks. Map-n-encap solutions use a mapping table to find out the destination network $N$ 's attachment point to the Internet core and encapsulate packets to $N$ with the attachment point's IP address, so that the packets can be forwarded across the network that does not have the specific routing entry for the destination network. Since mobility support solutions typically involve mapping mobiles' identifiers to the IP addresses of their current locations, there has been a reoccurring question on whether one could deploy one solution to solve both mobility support and global routing scalability problems. The recent effort to extend LISP to support mobility represents one attempt in this direction [12].

To answer this question, one needs to understand the different roles that mapping and encapsulation play for mobility and routing purposes. The fundamental difference between the two is that, in the context of MIP, all IP addresses are reachable, but a correspondent does not know (is not aware of) the current address of the mobile. Thus the correspondent sends packets to the mobile's Home Address, and the Home Agent, which holds the mapping information, intercepts and encapsulates the packets to reach the mobile. The Home Agent keeps track of the mapping information, but does not distribute it anywhere; the encapsulation is simply to preserve the sender identity.

In the context of map-n-encap based scalable routing designs, on the other hand, destination network prefixes are removed from the global routing table. Instead there must be a mapping table that contains each destination network's attachment points to the global Internet. Network entry point routers (a.k.a. "correspondents") need to know where the destinations are attached to, in order to encapsulate packets towards the right network exit points. Therefore, the mapping information must be made available at all the edge routers, so that they can properly encapsulate packets to reach the exit router for each destination network.
The mapping information can be either pushed to, or polled by, the network entry nodes, nevertheless the specific distribution mechanisms do not change the basic requirement that this information must be available at a large number of places.

If one added mobility support to the same mapping system used for routing scalability, it implies that mapping information must be distributed whenever movement occurs to assure data delivery. However, as we discussed earlier, when the network is large or when the number of mobiles is large, it is infeasible to distribute the mapping information widely. The recent effort to extend LISP to support mobility avoids the scalability concerns by making network entry point routers request the mapping between mobiles identifiers and IP addresses, and then cache the information, however no scalable way is found to flush out stale cache entries when mobiles move.

\section{THE ROAD TO FUTURE MOBILITY SUP- PORT}

In the last section we examined the fundamental differences between the mobility service models of cellular networks and Internet. We now turn our attention to identify both new opportunities and remaining open issues in providing global scale mobility support for unlimited number of online mobile devices on Internet.

\subsection{Open Platform Mobile Devices}

Up until now, cellphones in general run on a closed platform. That is, cellular network operators have the complete control over which handsets and wireless devices may connect to their networks, and what software to install on them. The launch of Apple's iPhone, as well as other smart-phones such as G-phone, announced a turning point in this traditional way of cellphone business. Although they are still being called a "phone", these smart phones are essentially a hand-held version of general purpose computers. The users can install any desired new applications to run on them; making phone calls is merely one of the many available applications. They usually have multiple network interfaces and can connect to other networks in addition to cellular networks. At any given moment these mobile device may simply want to get connected through whichever cheapest means they can find available, and this provides another strong argument for separating access control form mobility management.

Foreseeing the forthcoming pervasive hand-held mobile devices, many recent efforts have gone into developing new context and location-aware applications. Multiple devices in the same vicinity can discover each other and exchange useful information (such as highway traffic congestion; some of such applications already exist). This kind of ah hoc interconnects blur the notion of network access. However with the current cellphone service model, users' connectivity is provided and controlled by different service providers. The existing cellular network infrastructure provides incentives for intra-provider communications and disincentive for interprovider communications. Such constraints do not help mobile devices fully exploit their potential applications.

This new kind of open platform, hand-held mobile devices are taking the control away from the mobile service providers and putting the control in the hand of their users. Anyone 
who designs future mobility support protocols should take this new requirement, or rather, this new opportunity into account.

\subsection{Open Issues in Mobile IP Support}

Due to the space limit we touch only a subset of the open issues in future mobility support. Perhaps the best known problem with the initial MIP design is its side effect of triangle routing as we mentioned in Section 3.2. No matter where a Home Agent is places, it can be far off the shortest path between a correspondent and a mobile. Many research and standardization efforts have been devoted to address this problem, resulting in a plethora of proposed solutions with increased complexity in mobility support. A conceptually simple solution to this problem is to place home agents in multiple locations, and allow each correspondent to send data to a nearest one to reach the mobile, so that the communication path through home agent would be close to the direct path between the correspondent and the mobile as more home agents are deployed. This is the basic idea behind Global HAHA's design. However Global HAHA requires mapping information synchronization among the multiple home agents, and a new open issue arises regarding how to achieve this synchronization in a scalable way with a large number of mobiles and home agents.

Global HAHA [10] represents an effort to mitigate the triangle routing problems at IP layer. In contrast, HIP and MobileMe solutions [4] avoid triangle routing entirely by using dynamic DNS to keep track of the current IP address of a mobile, so that correspondents can use the addresses to communicate with mobiles directly. At this time we are not aware of large scale HIP deployment for mobility support; MobileMe has been a successful service over the last two years. However the mobility support of MobileMe follows a model of "resume after movement" which is adequate for the current applications supported by MobileMe, rather than communicating-while-moving model in cellular networks. At this early stage, it seems that the jury is still out regarding whether dynamic DNS can provide good enough rendezvous point function for communicating on the move applications.

Yet two other open issues are the security consideration and the overhead in mobility management. Securing mobile movement updates, which may be sent to either Home Agents or DNS servers, is an essential requirement, but the coordination between security mechanisms (e.g. IPsec and IKE) and mobility protocols introduces additional complexity [13]. The tradeoff between a solution's security strength and its practical performance must be taken into account when one designs global mobility services. Moreover, a mobile's fast movements can potentially lead to both high overhead and poor performance, as movement updates must be sent frequently, yet an update may still become obsolete when it reaches the Home Agent. To reduce the overhead and improve data delivery quality under fast movement, a number of effort have gone into localized mobility support, i.e. handling fast movement within a small scope, and updating the mapping at home agent only for big movements. Within a scope that is small enough, routing-based mobility support could also become feasible. It seems clear that such localized mobility support should be hidden from home agents, but it is unclear how much a mobile may need to participate in the localized mobility support.

\section{SUMMARY}

In this paper we presented a high level assessment on the current state of art and general direction for mobility support in the Internet. We put forth a position that network access control should not be coupled with mobility management, and that mobility support should be designed outside of the global routing system. We argued that decoupling network access from mobility management will enable different solutions for each of the two and facilitate innovations in future mobility applications.

This paper is not meant to suggest mobile providers to change their service models. Rather, the goal is to share the our articulation about the general direction for future mobility support to initiate an open discussion. We believe that we are at an early stage in understanding mobility support; the future offers a great potential with a high degree of uncertainty. It is possible, or even likely, that mobility support will be provided in multiple forms by multiple parties, perhaps with each tailored to different mobile devices and applications.

\section{REFERENCES}

[1] R. Bagrodia, M. Gerla, S. Lu, D. Valentino, L. Zhang. iMASH: Adaptive Middelware and Networking Support for the Nomadic Healer. Research proposal, August, 1999.

[2] D. Clark. The Design Philosophy of the DARPA Internet Protocols. SIGCOMM., 1988.

[3] A. L. Dul. Global IP Network Mobility using Border Gateway Protocol(BGP). Boeing White paper., 2006.

[4] S. Cheshire, L. Zhang What to Use as Host Identifier: there is theory, and there is practice. http://www.dagstuhl.de/Materials/Files/09/ 09102/09102.ZhangLixia1.Slides1.pdf, March, 2009.

[5] S. Gundavelli, K. Leung, V. Devarapalli, K. CHowdhury, B. Patil. Proxy Mobile IPv6 http://www.ietf.org/rfc/rfc5213.txt, 2008.

[6] R. Moskowitz, P. Nikander, P. Jokela, T. Henderson. Host Identity Protocol http://www.ietf.org/rfc/rfc5201.txt, 2008.

[7] J. Laganier, L. Eggert. Host Identity Protocol (HIP) Rendezvous Extension http://www.ietf.org/rfc/rfc5204.txt, 2008.

[8] R. Hinden. New Scheme for Internet Routing and Addressing (ENCAPS) for IPNG. RFC 1955, http://www.ietf.org/rfc/rfc1955.txt,1996.

[9] Xin Hu, Li Li, Mao, Z.M, Yang, Y.R. Wide-Area IP Network Mobility. INFOCOM, 2008.

[10] R. Wakikawa, G. Valadon, J. Murai. Migrating Home Agents Towards Internet-scale Mobility Deployments CoNEXT, 2006.

[11] Alcatel Lucent Introduction to Evloved Packet Core Strategic White Paper, 2009

[12] D. Farinacci, V. Fuller, D. Lewis, D. Meyer. LISP Mobility Architecture, http://www.ietf.org/id/draft-meyer-lisp-mn-00.txt, July 2009.

[13] B. Patil, D. Premec, C. Perkins, H. Tschofenig. Problems with the use of IPsec as the security protocol for Mobile IPv6. http://www.ietf.org/id/draft-patil-mextmip6issueswithipsec-01.txt, July 2009

[14] Host Identity Protocol Working Group, http://www.ietf.org/dyn/wg/charter/hip-charter.html

[15] Internet Research Task Force Routing Research Group, http://trac.tools.ietf.org/group/irtf/trac/wiki/ RoutingResearchGroup 